CORRECTION

https://doi.org/10.1038/s41586-019-1378-x

\title{
Author Correction: Diverse and robust molecular algorithms using reprogrammable DNA self-assembly
}

Damien Woods, David Doty, Cameron Myhrvold, Joy Hui, Felix Zhou, Peng Yin \& Erik Winfree

Correction to: Nature https://doi.org/10.1038/s41586-019-1014-9, published online 20 March 2019.

In Fig. 1 of this Letter, prime symbols were erroneously included in some labels in panels $\mathbf{c}$ and $\mathbf{d}$. In the bottom section of panel $\mathbf{c}$, in the diagram beneath 'SST self-assembly', the labels w2a', w3a', w4a' and w5a' should read w2a, w3a, w4a and w5a, respectively. Similarly, in panel d, the labels w $2 a^{\prime}$ and $w 3 a^{\prime}$ should read w2a and w3a, respectively. Additionally, there were some omissions in the Acknowledgements: R. Schulman should have been thanked for experimental advice, and R. Hariadi for contributing to preliminary designs for algorithmic self-assembly by SST. Finally, in Supplementary Figs. 8 and 9, the rightmost labels s should read s', and on page 64 of the Supplementary Information a citation to Telser et al. (1989) was missing and has been added as ref. 89; the subsequent citations have been renumbered. The Supplementary Information has been updated accordingly, and minor changes have also been made to the phrasing throughout to improve clarity. The original, incorrect version of the Supplementary Information is included as Supplementary Information to this Amendment, for transparency. The original Letter has been corrected online.

Supplementary Information is available in the online version of this Amendment. 